\title{
Recognition of Rotating Images Using an Automatic Feature Extraction Technique and Neural Networks
}

\author{
Brijesh Verma, Member IEEE \& IASTED \\ Griffith University-Gold Coast Campus \\ Faculty of Engineering and Applied Science \\ Parklands Dr. Gold Coast Qld 4217 Australia \\ E-mail: easbrij@ipnet.ins.gu.edu.au
}

\begin{abstract}
This paper presents a new automatic feature extraction technique and a neural network based classification method for recognition of rotating images. Image processing technique extracts global features of a image and converts a large size image into a one-dimensional small vector. An especial advantage of the proposed technique is that the extracted features are same even if original image is rotated with rotation angles from 5 to 355 or rotated and little bit distorted. The proposed technique is based on simple Co-ordinate geometry, fuzzy sets and neural networks. The proposed approach is very easy in implementation and it has implemented in $\mathrm{C}^{+}+$on Sun workstation. The experimental results have demonstrated that the proposed approach performs successfully on a variety of small as well as large scale rotated and distorted images.
\end{abstract}

\section{INTRODUCTION}

Image Processing techniques which are invariant to rotation and distortion and decrease the image size can be very useful for a variety of neural network based automated pattern recognition systems.

Image fragmentation, Fourier-Transformation and feature extraction by segmentation $[1,3,4,6,10]$ are often used to convert large size data sets into smaller before they can be used by Artificial Neural Networks (ANNs) because if the training data sets are smaller, ANNs training as well as recognition process is faster and free from many problems $[1,2]$.

A number of image processing and feature extraction algorithms have been proposed for feature extraction for Chinese character [11], Arabic character [5], Hindi character [1] and other images [4, 7] but most of the existing feature extraction techniques are rotation and distortion dependent and it is very difficult to extract features such as strokes, holes, endpoints, etc., if the image is little bit rotated, shifted or distorted.

In this paper we propose an approach for global feature extraction which is independent of rotation and distortion as well as converts two-dimensional large data set into one-dimensional data set and a neural network for classification of extracted features. The proposed approach is based on basics of Co-ordinate geometry, Fuzzy Sets [6] and ANNs [1, 2].

The remainder of this paper is organised as follows. In Section II, we present a full description of the proposed approach. Some experimental results are shown in Section III. Finally, Section IV concludes the paper.

\section{PROPOSED TECHNIQUE}

The proposed technique is based on the idea that each point on a circle (excluding inside or outside of a circle) has static distance from its centroid and it is called radius ( $x$ ). A point can be moved anywhere on a circle but distance $r$ from its centroid will not change and number of points is also static and it will not change when points are moved on a circle. Of course the number of points will be changed if we do not have a complete circle and it is a case of distorted images. One of the main roles of fuzzy techniques which we use in our approach, is to make complete circle from incomplete circle. Finally, we use feedforward neural network [1, 2] for classification of data collected from previous stage. The whole idea is described below. 


\subsection{Preprocessing}

We convert scanned image into digitised (binary) image ie., image with pixels 0 (white) and l(black). After converting image, we reduce unnecessary pixels $(0)$ from the original image. For this purpose, we use following algorithm.

\section{Algorithm 1:}

- Start from top-left corner, repeat for each column and row.

If (sum of all pixels) in column/row $>0$ then save column and row else don't save (delete from the image) column/row

- Calculate and save height and width of the reduced binary image

The whole process is shown in Figure 1.

\section{Centre of the Preprocessed Image}

We calculate the centre of the preprocessed image using formula as below.

$$
\text { Centre } x=\frac{\text { Width }+1}{2} ; \quad \text { Centre } y=\frac{\text { Height }+1}{2}
$$

Once we get the centre of the preprocessed image, we draw circles with centroid (centre $x$, centre $y$ ) and various radiuses (r_max, ... , r_min) as shown in Figure 4 and calculate the sum of all black pixels (1) as well as all black and white pixels (total pixels) belonging to each circle. This sum is fuzzy for distorted images. We use fuzzy sets with membership function as shown in Figure 5 and we calculate the membership values for each radius and those values are used by ANNs for final classification. For this purpose we use following algorithm.

Algorithm 2:

- For each black pixel $(x, y)$, calculate radius (r) using following formula.

$$
r=\sqrt{(\text { centre_ } x-x)^{2}}+(\text { centre_ } y-y)^{2}
$$

- Sum all pixels with same radius (sum_r_max, ... , sum_r_min).

- Calculate membership for each circle or disk using following membership function.

$$
m_{-} f(b p)=e^{-\frac{(\mid b p-r b p \|)}{\text { totat_pixels }}}
$$

where tbp $=($ total-pixel $)$ div 2 , total pixel $=($ black + white $)$ pixels belong to circle

\section{EXPERIMENTAL RESULTS}

The proposed approach has been implemented in $\mathrm{C}++$ on Sun workstation. Many images including medical, letters and faces were scanned and processed. The results are illustrated in Figures 1 and 2 . The original images were rotated and distorted and then proposed approach was used. In each case, global extracted features were same for same image in the form of a one-dimensional vectors. We provided those global features to the Neural Network for classification.

We used single hidden layer FNN and EBUDS [2] training algorithm. We scanned more than 100 images such as faces, mammograms and letters, some samples are presented in this paper. We extracted features using new technique and neural networks were trained using different inputs (faces, mammograms, letters) and 10 (coded eg., $4=0000000100$ ) outputs. Neural network classified all images correctly. The results for different images are shown in Table 1. 


\title{
3.1 EXPERIMENT 1
}

\author{
ORIGINAL IMAGE REDUCED (0) BINARY IMAGE
}

(s)

ORIGINAL BINARY IMAGE

$000000000000000000000000 \quad 0000000000011110000000$ $000000000000000000000000 \quad 0000000010111111100000$ $000000000000111100000000 \quad 000011111111111100000$ 000000000101111111000000 0001111111111111110000 00000111111111111000000 0011111111111111111000 00001111111111111100000011111111111111111100 0001111111111111110000 0111111111111111111110 00111111111111111111000 011111111111111111110 001111111111111111100 11111111111111111111 001111111111111111100 O11111111111101111110 $0111111111111111111110 \quad 11111111111000001110$ 0111111111111011111100 1111111110110000011111 01111111111100000711100 1111100111000000001110 $011111111101100000111110 \quad 1111100001100000011110$ $011111001110000000011100 \quad 1111000001100001101110$ $011111000011000000111100 \quad 1111000111000010111110$ $011110000011000011011100 \quad 1011000000000000000110$ $011110001110000101111100 \quad 1001000000000000000110$ $010110000000000000001100 \quad 1000000000000000000110$ $010010000000000000001100 \quad 1001000000000001000110$ $010000000000000000001100 \quad 1100100000000000001110$ $010010000000000010001100 \quad 11111000000000000001110$ $011001000000000001011100 \quad 1111100000011111011110$ $011111000000000000011100 \quad 1111110000000000011100$ $011111000000111110111100 \quad 1111101000000000111100$ 011111100000000000111000 P111100100000000111000 $011111100000000000111000 \quad 111110010000000111000$

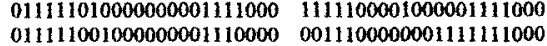
$011111000010000011110000 \quad 0000000000000011111100$ $000111000000011111110000 \quad 0000000000000000001000$ 00000000000000111111000

000000000000000000010000 000000000000000000000000 000000000000000000000000 000000000000000000000000 000000000000000000000000

HIEGHT $=30$; WIDTH $=22$; CENTRE $X=11.5 ;$ CENTRE $Y=15.5$;

RADIUSES:

$16.32515 .95315 .57215 .44315 .1161498314 .91614 .91614 .84914 .714 \quad 14.57714 .57714 .57714 .57714 .57714 .50914 .30014 .23014 .230$

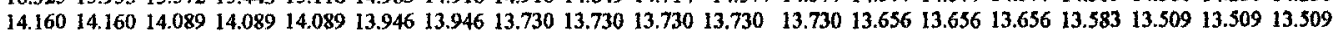

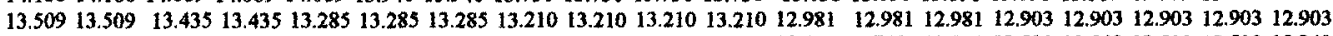

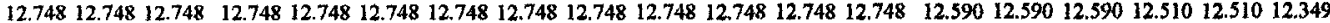
$12.34912 .34912 .34912 .349 \quad 12.34912 .34912 .34912 .34912 .34912 .10412 .10412 .10412 .10412 .10412 .10412 .10412 .10412 .02112 .021$ $12.02112 .02112 .02112 .02111 .853 \quad 11.85311 .85311 .85311 .85311 .76911 .76911 .59711 .59711 .59711 .51111 .51111 .51111 .51111 .511$ 11.51111 .5111 .51111 .51111 .51111 .42411 .42411 .42411 .42411 .33611 .33611 .33611 .33611 .33611 .33611 .33611 .33611 .06811 .06811 .068 11.511 11.511 11.511 11.511 11.511 11.42411 .42411 .42411 .42411 .33611 .33611 .33611 .33611 .33611 .33611 .33611 .33611 .06811 .06811 .068
$11.06811 .06811 .06810 .97710 .97710 .97710 .97710 .97710 .977 \quad 10.97710 .79410 .79410 .79410 .79410 .70010 .70010 .70010 .70010 .70010 .700$

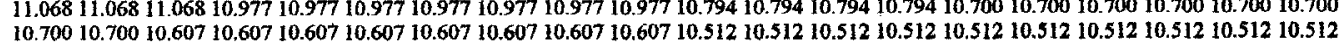
10.70010 .70010 .60710 .60710 .60710 .60710 .60710 .60710 .60710 .60710 .51210 .51210 .51210 .51210 .51210 .51210 .51210 .51210 .51210 .512 10.12410 .12410 .12410 .12410 .12410 .12410 .12410 .12410 .12410 .12410 .1249 .9259 .9259 .9259 .9259 .9259 .9259 .9259 .9259 .8239 .823
9.8239 .8239 .8239 .6189 .6189 .6189 .6189 .6189 .6189 .6189 .6189 .6189 .6189 .5139 .5139 .5139 .5139 .5139 .5139 .3019 .3019 .3019 .3019 .301

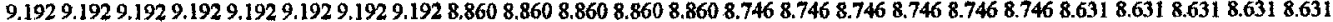
8.6318 .5158 .5158 .51585158 .5158 .5158 .5158515851585158 .5158 .2768776877687768 .2767 .9067 .9067906790679067 .9067 .906

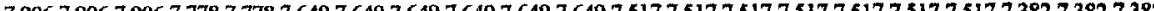

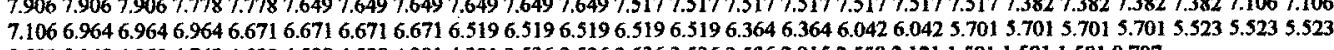
5.5235 .1484 .9504 .7434 .5284 .5284 .5284 .3014 .3013 .5363 .5363 .5363 .5363 .5362 .9152 .5502 .1211 .5811 .5811 .5810 .707 BLACK PLXES:

11111121151122325315234351332108652310486748810118510658566115102674334522441113251 1131

WHTTE PIXELS

444447344343433633733261231372113312223212421446536234322536221455411261247375687 773531

INPUT OF ANN (final one dimencionelil veetor):

$0.8190 .8190 .8190 .8190 .8190 .7790 .8190 .8190 .8190 .607 \quad 0.8191 .0001 .0000 .8190 .7170 .8820 .6070 .7170 .7790 .6070 .8190 .670$ $0.905 \quad 0.4720 .565 \quad 0.4720 .4720 .8190 .6620 .497 \quad 0.4240 .4350 .8190 .7170 .403 \quad 0.607 \quad 0.497 \quad 0.3350 .5490 .6070 .411 \quad 0.4970 .5650 .463$

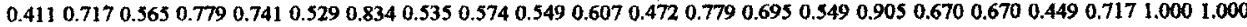
$0.7790 .936 \quad 1.0000 .7510 .6810 .8670 .8950 .6070 .7790 .8460 .7510 .9130 .7790 .6510 .6510 .7170 .6700 .717$

Figure 1. Results using proposed approach for original image. 


\title{
3.2 EXPERIMENT 2
}

\section{ORMGINAL ROTATED IMAGE REDUCED (0) BINARY IMAGE}

\author{
8 \\ ORIGINAL RINARY IMAGE \\ 0000000000000000000000000000000000000000001000 \\ $000000000000000000000000 \quad 0000000000000011111100$ \\ $000000000000000000000000 \quad 0011100000001111111000$ \\ $000000000000000000000000 \quad 1111100001000001111000$ \\ $000000000000000000010000 \quad 1111100100000000111000$ \\ $000000000000111111000-111110100000000111100$ \\ $00000000000000111111000 \quad 111101000000000111100$ \\ $000111000000011111110000 \quad 1111110000000000011100$ \\ $011111000010000011110000 \quad 1111100000011111011110$ \\ $011111001000000001110000 \quad 1111100000000000001110$ \\ $011111010000000001111000 \quad 1100100000000000001110$ \\ $011111100000000000111000 \quad 1001000000000001000110$ \\ $011111000000111110111100 \quad 1000000000000000000110$ \\ $011111000000000000011100 \quad 1001000000000000000110$ \\ $011001000000000000011100 \quad 10110000000000000000110$ \\ $010010000000000010001100 \quad 1111000111000010111110$ \\ $010000000000000000001100 \quad 1111000001100001101110$ \\ $010010000000000000001100 \quad 1111100001100000011110$ \\ $010110000000000000001100 \quad 1111100111000000001110$ \\ $\begin{array}{lll}011110001110000101111100 & 1111111110110000011111 \\ 01111000001100001101100 & 111111111110000011110\end{array}$ \\ 0111000001100001111100
01111000011000000111100
11111111111111101111110

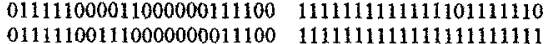 \\ 01111111101100000111110 0111111111111111111110 \\ 011111111111100000111100 0111111111111111111110 \\ $011111111111111011111100 \quad 0111111111111111111100$ \\ $01111111111111111111110 \quad 001111111111111111000$ \\ 00111111111111111111100 0001111111111111110000 \\ $\begin{array}{ll}001111111111111111111100 & 0001111111111111110000 \\ 00111111111111111111100 & 0000111111111111100000\end{array}$ \\ $0011111111111111111000 \quad 0000000010111111100000$ \\ $0001111111111111110000 \quad 0000000000011110000000$ \\ 00001111111111111100000 \\ 000001111111111111000000 \\ 000000000101111111000000 \\ 000000000000111100000000 \\ 0000000000000000000000000 \\ 000000000000000000000000 \\ HIEGHT $=30$; WIDTH=22; CENTRE_ $X=11.5 ;$ CENTRE_ $X=15.5$;
}

RADIUSES:

16.32915 .95315 .57215 .44315 .11614 .98314916149161484914 .7141457714577145771457714 .57714 .5091430014 .230

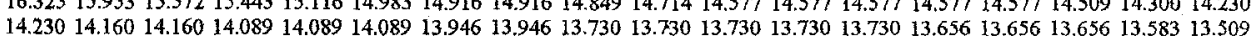

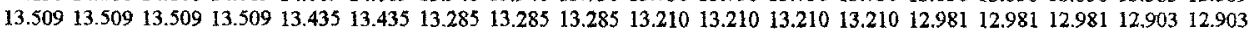

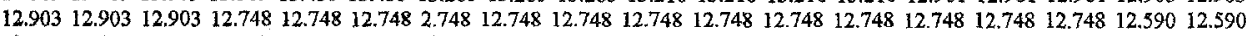
$12.59012 .51012 .51012 .34912 .34912 .34912 .34912 .34912 .34912 .34912 .349 .12 .34912 .349 \quad 12.104 \quad 12.104 \quad 12.10412 .10412 .104$ $12.10412 .10412 .10412 .02112 .02112 .02112 .02112 .02112 .02111 .853 \quad 11.85311 .85311 .85311 .853 \quad 11.76911 .76911 .59711 .597$ $11.59711 .51111 .51111 .51111 .51111 .51111 .51111 .51111 .511 \quad 11.51111 .51111 .42411 .42411 .42411 .42411 .33611 .33611 .336$ 11.3361133611 .33611336113361106811068110681106811 .0681106810 .97710 .97710 .9770 .97710 .97710 .97710 .977 $10.79410 .79410 .79410 .79410 .70010 .70010 .70010 .70010 .70010 .70010 .70010 .70010 .607 \quad 10.60710 .607 \quad 10.6070 .60710 .607$ $10.60710 .60710 .51210 .51210 .51210 .512 \quad 10.51210 .51210 .51210 .51210 .51210 .51210 .12410 .124 \quad 10.12410 .12410 .12410 .124$ 10.12410 .12410 .124 10.124 10.124 9.925 9.925 9.925 9.925 9.925 9.925 9.925 9.925 9.823 9.823 9.823 9.823 9.823 9.618 9.618 9.618

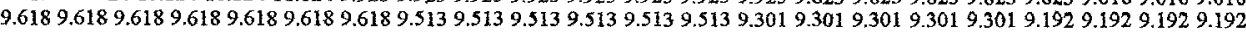
9.1929 .1929 .1929 .1928 .8608 .8608 .8608 .8608 .8608 .7468 .7468 .7468 .7468 .7468 .7468 .6318 .6318 .6318 .6318 .6318 .6318 .515 8.515 8.515 8.515 8.515 8.515 8.515 8.515 8.515 8.515 8.515 8.276 8.276 8.276 8.276 8.276 7.906 7.906 7.906 7.906 7.906 7.906 7.906 7.906 7.906 7.906 7.778 7.778 7.649 7.649 7.649 7.649 7.649 7.649 7.517 7.517 7.517 7.517 7.517 7.517 7.517 7.382 7.382 7.382 7.382 7.1067 .1067 .1066 .9646 .96469646 .67166716 .6716 .67165196 .51965196 .51965196364636460426 .0425 .7015 .7015 .701 3.701 5.523 5.523 5.523 5.523 5.1484.950 4.7434.528 4.528 4.5284.3014.3013.5363.536 3.536 3.5363.5362.9152.550 2.121 1.581 3.7015 .5235 .523
1.5811 .5810 .707 BLACK PIXELS:

11111121151122325315234351332108652310486748810118510658566115102674334522441 1132511131

WHITE PIXELS:

4444473443434336337332612313721133122232124214465362343225362214554112612473 75687773531

INPUT OF ANN (final one dimensional vector):

0.8190 .8190 .8190 .8190 .8190 .7790 .8190 .8190 .8190 .6070 .8191 .0001 .0000 .8190 .7170 .8820 .6070 .7170 .7790 .6070 .8190 .670

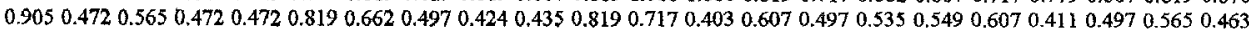

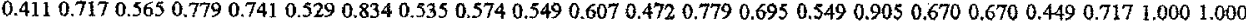
0.7790 .9361 .0000 .7510 .6810 .8670 .8950 .6070 .7790 .8460 .7510 .9130 .7790 .6510 .6510 .7170 .6700 .717

Figure 2, Results using proposed approach for rotated image. 
Recognition of Rotating Images Using an Automatic Feature Extraction Technique and Neural Networks

\section{ORIGINAL IMAGE}

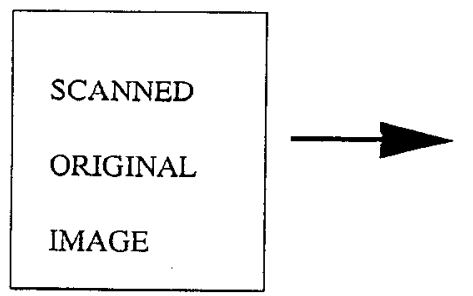

BINARY IMAGE

000000000000000 000000000000000

011100000111000 011111111111000 000000000011000 011111111111000 000000000000000

\section{REDUCED BINARY IMAGE}

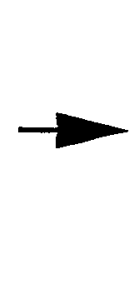

11100000111

11111111111

00000000011

11111111111

Figure 3. Original, binary and reduced binary images.
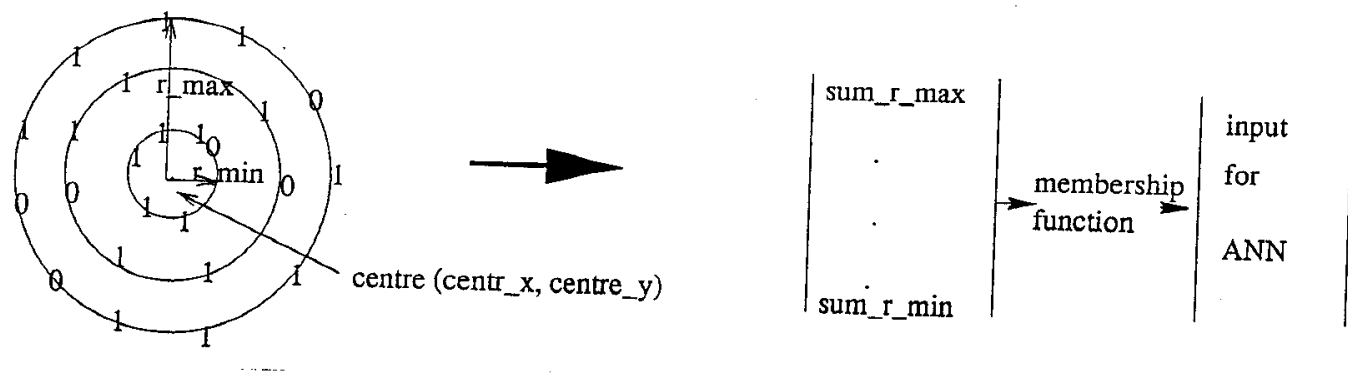

Figure 4. Preprocessed image with circles (centroid and radiuses).

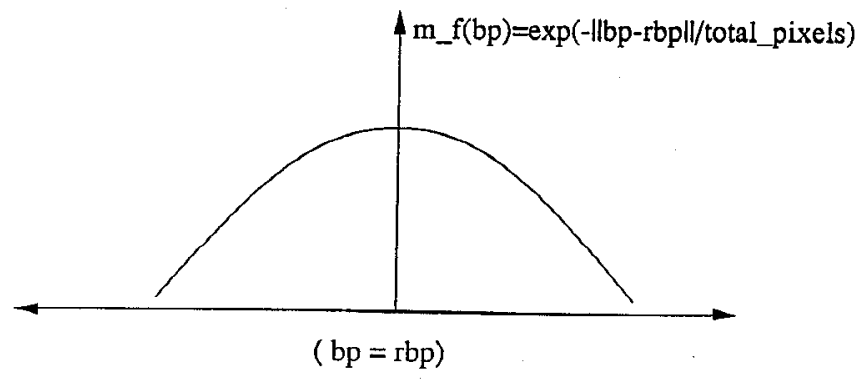

Figure 5. Membership function. 


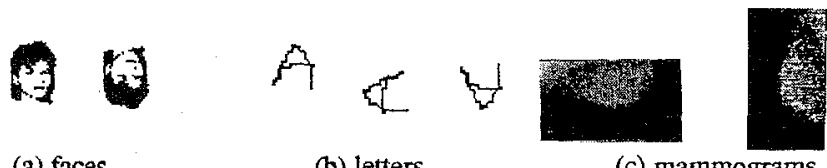
(a) faces
(b) letters
(c) marnmograms

Some samples of rotated images used for experiments

\begin{tabular}{|c|c|c|c|}
\hline & Input Images & \# of Images & Classification Rates [\%] \\
\hline 1. & Faces $(24 \times 36)$ & 50 & 100 \\
\hline 2. & Mammograms $(2048 \times 2048)$ & 20 & 100 \\
\hline 3. & Letters $(24 \times 40)$ & 50 & 100 \\
\hline
\end{tabular}

Table 1: Classification rates for different images with rotation and distortion using neutral networks

\section{CONCLUSIONS}

We have presented an approach for image recognition which is independent of rotation and converts a large scale image data into a small one-dimensionai vector with global features. Those extracted features are used by neural networks for classification of images. We have tested new technique using different images and results were promissing. The tesults for classification of different rotated and distorted images by neural network are presented in Table 1.

The presented technique has proven to be very effective at recognising a wide variety of images regardless of orientation. A small one-dimensional data from large scale image data which we get using proposed techniques, avoids the computer memory-problem, long training time in learning and recognition processes and neural network has guaranteed training, better generalisation and recognition accuracy.

\section{REFERENCES}

[1]. B.K. Verma, "Handwritten hindi character recognition using MLP and RBF neural networks," in Proc. of IEEE International Conference on Neural Networks, ICNN'95, Perth, November 1995.

[2]. B.K. Verma and J.J. Mulawka "A Modified Backpropagation Algorithm," in Proc. of IEEE International Conference on Neural Networks, ICNN'94, Orlando, June-July 1994.

[3]. D. Flotzinger and M. Pregemer, "Feature selection with distinction sensitive learning vector quantisation and genetic algorithms" In Proc. of IEEE International Conference on Neural Networks,ICNN'94, pp. 3448-3452, Orlando, June-July 1994.

[4]. E. Cohen and J.J. Hull, "Control structure for interpreting handwritten addresses," IEEE Trans. on Pattern Analysis and Machine Intelligence, vol. 16, no. 10, October 1994.

[5]. I.S. Abuhaiba and S.A Mahmoud, "Recognition of handwritten cursive arabic characters," IEEE Trans. on Pattern Analysis and Machine Intelligence, vol. 16, no. 6, June 1994.

[6]. J. Bezdek, "Fuzzy models what are they, and why," IEEE Transaction on Fuzzy Systems, yol. 1, no, 1, pp. $1-5,1993$

[7]. J.H. Chia-Lun, "Fourier-Transformed preprocessing used in a noniteratively-trained perceptron pattern recognizer," in Proc. of IEEE International Conference on Neural Networks, ICNN'94, pp. 3020-3023, Orlando, June-July 1994.

[8]. J.L. Hemminger, "Using a hopfield network for rotation and scale independent pattern recognition," in Proc. of IEEE International Conference on Neural Networks, ICNN'94, pp. 3003-3007, Oriando, JuneJuly 1994.

[9]. J.S. Roger, "Functional equivalence between radial basis function networks and fizzy inference systems, IEEE Transaction on Neural Networks," vol. 4, no. 1, pp. 156-158, 1993.

[10]. J.M. Koch and M. Moya, "Feature discovery in gray level imagery for one-class object recognition," in Proc. of IEEE International Conference on Neural Networks, ICNN'94, pp. 2979-3451, Oriando,June-July 1994.

[11]. X. Huary and J. Gu, "A constrained approach to muitifont chinese character recognition," IEEE Trans. on Pattern Analysis and Machine Intelligence, vol. 15, no. 8, August 1993. 